\title{
Detection of surface carbon and hydrocarbons in hot spot regions of niobium superconducting rf cavities by Raman spectroscopy
}

\author{
C. Cao, ${ }^{1,2}$ D. Ford ${ }^{3,4}$ S. Bishnoi, ${ }^{1}$ T. Proslier, ${ }^{2}$ B. Albee, ${ }^{1, *}$ E. Hommerding, ${ }^{1}$ A. Korczakowski, ${ }^{1}$ L. Cooley, ${ }^{3}$ \\ G. Ciovati, ${ }^{5}$ and J.F. Zasadzinski ${ }^{1,2, \dagger}$ \\ ${ }^{1}$ Physics Department, Illinois Institute of Technology, Chicago, Illinois 60616, USA \\ ${ }^{2}$ Materials Science Division, Argonne National Laboratory, Argonne, Illinois 60439, USA \\ ${ }^{3}$ Superconducting Materials Department, Technical Division, Fermi National Accelerator Laboratory, \\ Batavia, Illinois 60510, USA \\ ${ }^{4}$ Department of Chemical and Biological Engineering, Northwestern University, Evanston, Illinois 60208, USA \\ ${ }^{5}$ Thomas Jefferson National Accelerator Facility, Newport News, Virginia 23606, USA
}

(Received 5 November 2012; published 26 June 2013)

\begin{abstract}
Raman microscopy/spectroscopy measurements are presented on high purity niobium (Nb) samples, including pieces from hot spot regions of a tested superconducting rf cavity that exhibit a high density of etch pits. Measured spectra are compared with density functional theory calculations of Raman-active, vibrational modes of possible surface $\mathrm{Nb}-\mathrm{O}$ and $\mathrm{Nb}-\mathrm{H}$ complexes. The Raman spectra inside particularly rough pits in all $\mathrm{Nb}$ samples show clear differences from surrounding areas, exhibiting enhanced intensity and sharp peaks. While some of the sharp peaks are consistent with calculated $\mathrm{NbH}$ and $\mathrm{NbH}_{2}$ modes, there is better overall agreement with $\mathrm{C}-\mathrm{H}$ modes in chain-type hydrocarbons. Other spectra reveal two broader peaks attributed to amorphous carbon. Niobium foils annealed to $>2000^{\circ} \mathrm{C}$ in high vacuum develop identical Raman peaks when subjected to cold working. Regions with enhanced $\mathrm{C}$ and $\mathrm{O}$ have also been found by SEM/EDX spectroscopy in the hot spot samples and cold-worked foils, corroborating the Raman results. Such regions with high concentrations of impurities are expected to suppress the local superconductivity and this may explain the correlation between hot spots in superconducting rf (SRF) cavities and the observation of a high density of surface pits. The origin of localized high carbon and hydrocarbon regions is unclear at present but it is suggested that particular processing steps in SRF cavity fabrication may be responsible.
\end{abstract}

DOI: 10.1103/PhysRevSTAB.16.064701

PACS numbers: 74.62.Bf, 74.62.Dh, 74.25.nd

\section{INTRODUCTION}

Superconducting radio frequency (SRF) cavities, having $\mathrm{Q}$ values orders of magnitude higher than normal $\mathrm{Cu}$, are an enabling device for a host of linear particle accelerator applications [1]. SRF cavities made from high purity $\mathrm{Nb}$ plates involve numerous processing steps including deep drawing, welding, acid etching/polishing, two types of vacuum annealing, and high-pressure water spraying. The connections between processing and the surface superconductivity relevant for cavity operation are still not well understood [2]. There is a need to develop new analytical techniques that can easily measure the material properties at the nanoscale, i.e., within a magnetic penetration depth, $\lambda \sim 45 \mathrm{~nm}$, of the surface. Here we show that Raman spectroscopy combined with density functional theory

\footnotetext{
*Currently at University of California-Irvine, Irvine, California 92697, USA.

Corresponding author. zasadzinski@iit.edu
}

Published by the American Physical Society under the terms of the Creative Commons Attribution 3.0 License. Further distribution of this work must maintain attribution to the author(s) and the published article's title, journal citation, and DOI.
(DFT) offers promise as a unique and direct approach to detecting trace levels of surface inclusions such as hydrides and hydrocarbons that may be playing a critical role in the performance of an SRF cavity.

Chemical polishing of the niobium surface is a vital step in the cavity processing procedure which reduces the surface roughness and removes the layer damaged from forming; however, it was recognized several decades ago that chemical contamination is a serious drawback to these procedures [3]. Numerous reports describe how cavities tested immediately after heavy chemical polishing developed a rapid drop of $\mathrm{Q}$ starting with zero accelerating gradient (so-called Q disease [4]), and the susceptibility of niobium to hydrogen absorption during chemical processing has been established [5]. Dissolved oxygen, nitrogen, and carbon are all found in SRF niobium [6], although specifications for the niobium typically restrict their concentrations to $<200$ atomic $\mathrm{ppm}$. The above-mentioned impurities are problematic for SRF cavities because they reduce the thermal conductivity of niobium, thereby reducing its ability to dissipate heat during rf operation [7]. Furthermore, dissolved oxygen [8] and hydrogen $[9,10]$ are known to decrease the superconducting transition temperature of niobium at concentrations of a few atomic percent. 
In addition to $\mathrm{Q}$ disease, impurities may affect a cavity's performance by Q drop, the reduction of Q with increasing rf fields, which is not yet fully understood. A particular case study of interest is a single-cell niobium SRF cavity, fabricated using a buffered chemical polishing (BCP), that exhibited medium field (20-100 mT) Q drop [11]. Localized regions of high dissipation, hot spots, developed as Q decreased, and optical and electron backscattered diffraction imaging showed clearly that such hot spots were correlated with a high areal density of etch pits, especially near grain boundaries. Cold spots had more shallow pits with significantly lower density. We might hypothesize therefore that the dissipation is related to different surface structure and chemistry inside or beneath the pits as compared to other regions of the cavity surface. It has been shown previously that pits are found in conjunction with high dislocation density $[12,13]$ and dislocations trap impurities [14]. However, a link between hot spots and the chemistry underlying the etch pits is not yet confirmed. Thus, we obtained several samples from the cavity of Ref. [11] to include in our study.

The standard spectroscopy tools of electron microscopy, namely Auger and energy dispersive x-ray (EDX), showed little difference between the regions inside and outside the pits in preliminary tests [11]. One concern is that such spectroscopic tools cannot measure hydrogen, a gas which diffuses easily in $\mathrm{Nb}$ and is a well-known contaminant potentially affecting the quality factor $\mathrm{Q}$ of the cavity [5]. Also, these techniques measure an average composition over a relatively large electron probing depth, $>100 \mathrm{~nm}$, and thus would be insensitive to subtle changes in surface oxide composition occurring over $\sim 5 \mathrm{~nm}$. This can be significant as it has been shown that $<3 \%$ oxygen vacancies in $\mathrm{Nb}_{2} \mathrm{O}_{5}$ change this compound from a nonmagnetic insulator to a conductor with localized magnetic moments [15]. The superconducting gap region probed by tunneling sometimes exhibits a smeared density of states consistent with pair breaking due to magnetic scattering [16]. There is thus a good chance that the source of rf dissipation in a pit is tied to the local chemistry (e.g. oxides, hydrides, dissolved gases, interstitial impurities, precipitates) near the surface, which might suppress the superconductivity in a variety of ways and cause increased rf impedance and dissipation.

Raman microscopy/spectroscopy is a technique which has not previously been used for Nb SRF cavity research. Raman is a simple, fast, generally nonperturbative optical method that probes molecular vibrational modes as well as bulk phonons via inelastic scattering. The probing depth in pure $\mathrm{Nb}(\sim 10-20 \mathrm{~nm})$ is estimated from the skin depth of the $785 \mathrm{~nm}$ laser and is a reasonable fraction of the magnetic penetration depth [2]. Since pure $\mathrm{Nb}$ itself is not Raman active, any backscattering signal must originate from surface oxides, dissolved species, or inclusions near the surface. Surface impurities might reduce the electrical conductivity and increase the Raman probing depth. Using a 50X objective lens, the laser spot size is $\sim 3 \mu \mathrm{m}$, which is ideal for examining etch pits that range in lateral dimension from $\sim 10 \mu \mathrm{m}$ to $\sim 200 \mu \mathrm{m}$. As will be shown, Raman spectra display striking differences between the inside and outside of certain types of etch pits. A principal discovery is the consistent observation of sharp Raman peaks inside these etch pits as well as in cold-worked regions of $\mathrm{Nb}$ foils. This indicates that the Raman peaks are linked to a high dislocation density. The DFT calculations show agreement of some peaks with hydride phases $\mathrm{NbH}$ and $\mathrm{NbH}_{2}$. However, the overall spectra are more consistent with the $\mathrm{C}-\mathrm{H}$ modes found in chainlike hydrocarbons [17]. Another group of spectra exhibit relatively broader peaks that are identified as amorphous carbon. A reexamination of the hot spot samples and $\mathrm{Nb}$ foils by SEM/EDX indeed showed regions with high $\mathrm{C}$ and $\mathrm{O}$ content. These results suggest that regions of high dislocation density may be trapping migrating $\mathrm{C}$ and $\mathrm{O}$ as well as dissolved hydrogen with sufficient concentration to form inclusions of stable compounds. However, there is also the possibility that stable hydrocarbons are already present in the $\mathrm{Nb}$ as a consequence of processing such as deep drawing. These results provide a reasonable explanation of the correlation of hot spots in SRF cavities with the observation of a high density of etch pits.

\section{EXPERIMENT}

All Raman measurements were done using a Renishaw, inVia, Raman microscope with a $785 \mathrm{~nm}$ laser source, $10 \mathrm{~s}$ exposure time, $100 \%$ laser power $(27 \mathrm{~mW})$, and a $50 \mathrm{X}$ objective lens. All SEM/EDX measurements were done using the Hitachi S-4700 FESEM and EDXS acquisition system, with an accelerating field of $10 \mathrm{kV}$ and an emission current of 10-20 $\mu \mathrm{A}$. The probing depth is $\sim 1 \mu \mathrm{m}$.

The types of $\mathrm{Nb}$ samples studied included cut-outs from a tested cavity as well as high purity $\mathrm{Nb}$ foils. One group of foils had been rolled from a piece of $\mathrm{Nb}$ plate used for $\mathrm{SRF}$ cavity fabrication and subsequently processed in a similar manner to that used on cavities including electropolishing and BCP etching [2,5,11]. In addition we examined foils as obtained directly from Alpha Aesar (as-received noted here as unprocessed). In some cases the foils were resistively heated near the melting point of $\mathrm{Nb}\left(2469^{\circ} \mathrm{C}\right)$ in high vacuum $\left(10^{-7}\right.$ torr $)$. These latter foils showed single crystal regions up to a few millimeters in diameter and are referred to as recrystallized. Several of these recrystallized foils were subsequently cold worked by bending back and forth $\left(\sim 90^{\circ}\right.$ angle) from 20 to 50 times. We also have Raman data from six different hot spot samples cut out of a single-cell cavity [11]. The hot spots displayed temperature rises $\sim 0.3-0.4 \mathrm{~K}$ above the He bath temperature at cavity magnetic field strengths of $90 \mathrm{mT}$. The cavity was made from a large-grain plate with a residual resistance ratio of 280 and was etched by BCP. Its performance was 
characterized by a strong medium field Q slope and no field emission. The detailed processing history and cavity performance measurements can be found in Ref. [11]. Sample numbers were those assigned at Jefferson Lab and are referred to as, for example, Jlab \#12.

All of the calculations were performed with the Vienna ab initio Simulation Package (VASP) [18,19], using DFT, periodic boundary conditions, and a plane wave basis set with a $400 \mathrm{eV}$ kinetic energy cutoff. The generalized gradient approximation was used with the Perdew, Burke, Ernzerhof [20] exchange-correlation functional, and the core electrons were described by the projectoraugmented-wave (PAW) [21,22] pseudopotentials. The geometry was optimized for each crystal structure with a 0.25 A gamma centered k-point mesh determined from the Monkhorst-Pack scheme [23]. The partial occupancies for the wave functions were set by the 1 st order Methfessel-Paxton method with a smearing width of $0.2 \mathrm{eV}$ for metallic structures and the tetrahedron with Blochl corrections for $\mathrm{Nb}_{2} \mathrm{O}_{5}$. The phonon spectra were calculated using the phonon module in the MEDEA software package. This program applies the direct method [24] for calculating vibrational frequencies and uses forces calculated with VASP. Supercells extending at least $10 \AA$ in each lattice direction were created to minimize the interactions between equivalent atoms in adjacent cells, and atoms were displaced $0.02 \AA$ from their equilibrium positions for the force calculations. The results are presented as the phonon density of states (DOS) at wave numbers where the gamma point frequency is Raman active. We call this the Raman DOS.

The niobium hydride structures were modeled as $\delta$ $\mathrm{NbH}_{2}$ (Fm-3 m, one formula unit per unit cell), $\beta \mathrm{NbH}$ (cccm, four formula units per unit cell), and $\eta \mathrm{Nb}_{4} \mathrm{H}_{3}$ ( $\mathrm{NbH}$ with one $\mathrm{H}$ removed from the unit cell containing four $\mathrm{Nb}$ and four $\mathrm{H}$ ). The $\alpha$ and $\alpha^{\prime}$ niobium hydride phases were modeled as body-centered cubic (bcc) niobium (16 atoms per unit cell) with three and eight hydrogen atoms, respectively, inserted into tetrahedral interstices in disordered configurations. The niobium oxide structures were modeled as $\mathrm{NbO}(\mathrm{Pm}-3 \mathrm{~m}$, three formula units per unit cell), $\mathrm{NbO}_{2}$ (14-1, eight formula units per unit cell), and $\mathrm{Nb}_{2} \mathrm{O}_{5}$ (P2, seven formula units per unit cell). Interstitial impurities in niobium were modeled in a $4 \times 4 \times 4$ bcc niobium lattice (128 Nb atoms). Experimentally determined lattice parameters for $\mathrm{Nb}$ [25], $\mathrm{NbH}$ [26], $\mathrm{NbH}_{2}$ [27], $\mathrm{NbO}$ [28], $\mathrm{NbO}_{2}$ [29], and $\mathrm{Nb}_{2} \mathrm{O}_{5}$ [30] are available in the literature and are $<1 \%$ different from our calculated values.

\section{RESULTS AND DISCUSSION}

To introduce our study we show, in the lower right hand panel of Fig. 1, a relatively wide-area optical microscope image of a region on hot spot \#9 that displayed a high density of etch pits. The region shown is characterized by

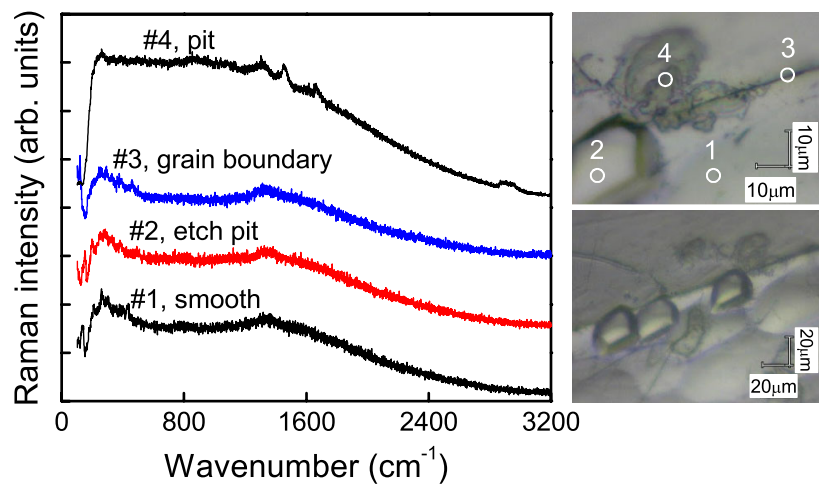

FIG. 1. The right panel shows two optical images of a region near grain boundary with high density of etch pits on Jlab \#9. The left panel shows corresponding Raman spectra of circled regions.

three etch pits $\sim 20 \mu \mathrm{m}$ on edge near a grain boundary. In addition, other rough patches of similar size to the etch pits are observed. An expanded view of the region near the topmost etch pit is shown in the upper panel of Fig. 1. Representative Raman spectra are shown for the four regions indicated. For smooth regions outside the etch pits (e.g. \#1), the Raman spectra are relatively flat and featureless showing only a broad peak near $1350 \mathrm{~cm}^{-1}$. Nearly identical Raman spectra are found in region \#2 which is inside a standard-type, faceted etch pit indicating no apparent differences chemically from surrounding regions as mentioned in Ref. [11]. Surprisingly, the grain boundary (\#3) shows little difference in the Raman spectra.

However, clear differences in Raman signal are found in regions of the type labeled \#4 which appear to be much rougher than surrounding areas, but still in the areas of high etch pit density. Atomic force microscopy images of those regions showed a higher rms surface roughness, typically by a factor of $\sim 10-20$. The Raman intensity is always enhanced, by as much as 50X (rescaled down for the figure), and there are well-defined sharp peaks in the region of $1000-1500 \mathrm{~cm}^{-1}$ and near $2900 \mathrm{~cm}^{-1}$. As will be shown, these peaks are quite reproducible, being observed in each of the six hot spot samples, as well as in coldworked $\mathrm{Nb}$ foils. In addition, there is another group of spectra characterized by two relatively broad peaks which are again consistently found on rough patches of hot spot samples as well as cold-worked foils. The main focus of the present work is the identification of these Raman peaks.

\section{A. Interstitial impurities}

We begin by presenting the DFT modeling results for interstitial impurities in bulk bcc niobium. Common impurities found in SRF niobium (hydrogen, nitrogen, carbon, and oxygen) vibrate at wave numbers below $1500 \mathrm{~cm}^{-1}$, as shown in Table I. Hydrogen is the only species that appears in the region of the peaks that we wish to identify in our measured Raman spectra. 
TABLE I. Vibrational modes of interstitial impurity atoms in the bcc niobium lattice from DFT calculations. $\mathrm{O}, \mathrm{N}$, and $\mathrm{C}$ are located in octahedral lattice interstices and $\mathrm{H}$ is in a tetrahedral lattice interstitial site.

\begin{tabular}{lcccr}
\hline \hline & $\mathrm{O}$ & $\mathrm{N}$ & $\mathrm{C}$ & $\mathrm{H}$ \\
\hline Mode 1 $\left(\mathrm{cm}^{-1}\right)$ & 307 & 423 & 489 & 968 \\
Mode 2 $\left(\mathrm{cm}^{-1}\right)$ & 698 & 759 & 806 & 1404 \\
\hline \hline
\end{tabular}

\section{B. Niobium oxide modes}

To test our methods and confirm that our measured Raman spectra are not due to the native oxide coating on niobium, we calculated the Raman DOS of $\mathrm{NbO}, \mathrm{NbO}_{2}$, and $\mathrm{Nb}_{2} \mathrm{O}_{5}$, and present the results in Fig. 2. $\mathrm{NbO}$ does not have any Raman-active modes. We also show our measured spectra for $\mathrm{NbO}_{2}$ and $\mathrm{Nb}_{2} \mathrm{O}_{5}$ powders as validation for the use of calculated Raman DOS for interpretation of Raman spectra [3]. Overall there is excellent agreement between the Raman DOS and $\mathrm{Nb}_{2} \mathrm{O}_{5}$ powder, indicating the accuracy of the VASP calculations. We note, however, that the intensities of the $\mathrm{Nb}-\mathrm{O}$ bending modes between $600-700 \mathrm{~cm}^{-1}$ and the double bond stretch mode near $980 \mathrm{~cm}^{-1}$ are higher in the Raman spectra of the powder than in the calculated Raman DOS, probably due to matrix element effects. Modes below $120 \mathrm{~cm}^{-1}$ are not recorded in the Raman measurement. The presence of the $980 \mathrm{~cm}^{-1}$ mode in the $\mathrm{NbO}_{2}$ powder indicates that oxidation of this powder has occurred after exposure to the air. This is further confirmed by the weak double peak between $600-700 \mathrm{~cm}^{-1}$, close to the two peaks of $\mathrm{Nb}_{2} \mathrm{O}_{5}$. Thus the $\mathrm{NbO}_{2}$ Raman spectrum has a significant contribution of $\mathrm{Nb}_{2} \mathrm{O}_{5}$ modes and is best interpreted as a mixture of the two oxides.

A comparison of Figs. 1 and 2 shows that $\mathrm{Nb}$ oxide modes are generally not detected on SRF cavity pieces

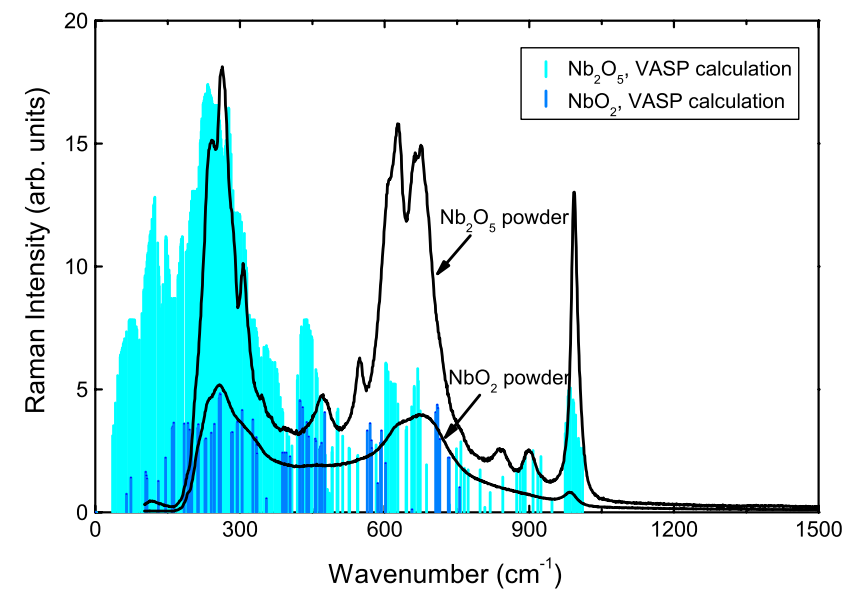

FIG. 2. Experimental Raman spectra of $\mathrm{Nb}_{2} \mathrm{O}_{5}$ and $\mathrm{NbO}_{2}$ powders (black lines) along with DFT calculations of the Raman DOS for $\mathrm{Nb}_{2} \mathrm{O}_{5}$ (light blue) and $\mathrm{NbO}_{2}$ (dark blue). or $\mathrm{Nb}$ foils. This is probably due to the oxide thickness $(\sim 5 \mathrm{~nm})$ giving a weak backscatter signal. However, oxide modes are clearly seen on $\mathrm{Nb}$ powders [31] where the effective surface for Raman backscatter can be considerably larger.

\section{Niobium hydride modes}

The calculation results for various $\mathrm{Nb}-\mathrm{H}$ complexes, including ordered hydride modes are shown in Fig. 3, along with the measured Raman spectra for a smooth and rough patch of an etched foil. It is clear that several of sharp Raman peaks observed inside pits or rough patches correlate with the phonon modes calculated for the $\mathrm{Nb}-\mathrm{H}$ complexes. In particular, the $\beta \mathrm{NbH}$ and $\delta \mathrm{NbH}_{2}$, which have the highest Raman DOS in the calculations, correlate with the strongest observed Raman peaks at 1068, 1303, and $1450 \mathrm{~cm}^{-1}$.

Since etch pits are a fingerprint of underlying crystal defects such as dislocations, it was hypothesized that the introduction of dislocations into a sample might generate such peaks. To test this hypothesis, several of the recrystallized foils were cold worked by bending back and forth to generate a high dislocation density. The sharp peaks typical of the Raman spectra inside of pits were reproduced throughout the cold-worked region as shown by a representative spectrum (top curve) in Fig. 3. This might suggest that the origin of the sharp Raman peaks is the trapping of impurities by dislocations present near the surface.

Similar experiments were performed on the Jlab hot spot samples. Regions viewed under the optical microscope of the Raman spectrometer appeared either as light or dark. Dark regions often corresponded to etch pits similar in dimension to those reported in Ref. [11]. An example of a pit from sample \#12 is shown in the photograph inset of

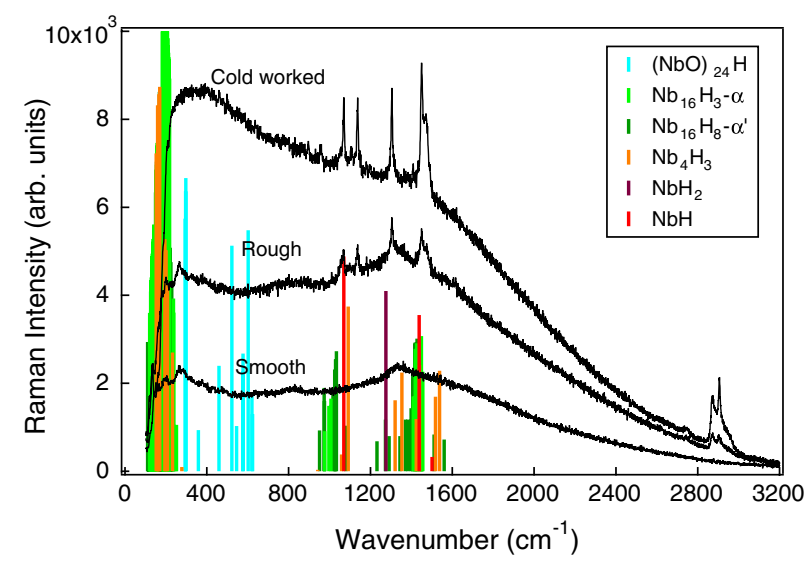

FIG. 3. Raman spectra from a recrystallized $\mathrm{Nb}$ foil. The rough spot is likely a pit and the smooth region is outside the pit. The cold-worked region has a spectrum similar to that inside the pit. Vertical lines are the DFT calculated Raman-active modes for various $\mathrm{Nb}-\mathrm{H}$ complexes as indicated in the inset legend. 


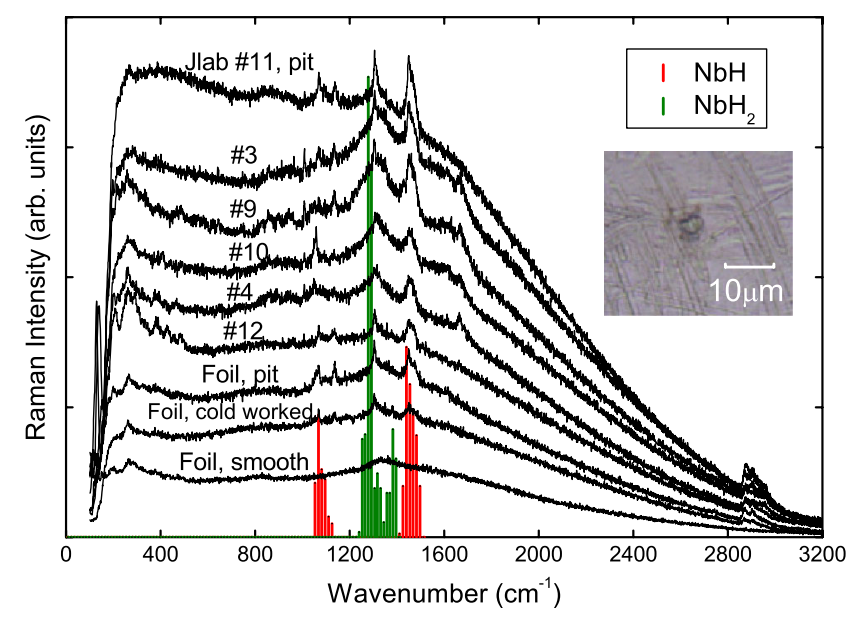

FIG. 4. Raman spectra inside pits or rough spots on six different hot spot samples. The dark spot on the inset shows the optical image of a pit. For comparison the data from a recrystallized $\mathrm{Nb}$ foil (pit and cold-worked region) are shown along with the typical spectrum from a smooth region. Red and green lines correspond to the partial DOS of the entire optical branch for $\mathrm{NbH}$ and $\mathrm{NbH}_{2}$, respectively.

Fig. 4. A significant fraction of the pits showed spectra similar to those found on $\mathrm{Nb}$ foils and a comparison of representative spectra is shown in Fig. 4. Each of the six hot spot samples is represented and similar spectra were observed in multiple pits on each sample. There is remarkable agreement among the spectra, which indicates that the development of particular impurity complexes in the subsurface of some pits is a rather common phenomenon.

Since hydride inclusions precipitating at a dislocation would likely be nanoscale, there could be significant phonon scattering which would generate disorder induced first order Raman scattering from the entire optical mode branch [32]. Considering this possibility, we compared the Raman spectra to the partial DOS of the optical branches that have a Raman-active mode at the zone center. These DOS calculations are shown as the red and green lines for $\mathrm{NbH}$ and $\mathrm{NbH}_{2}$, respectively. The agreement in location and characteristic width of the calculated and measured Raman peaks is quite good and the interpretation that these were hydride phases was made in an earlier

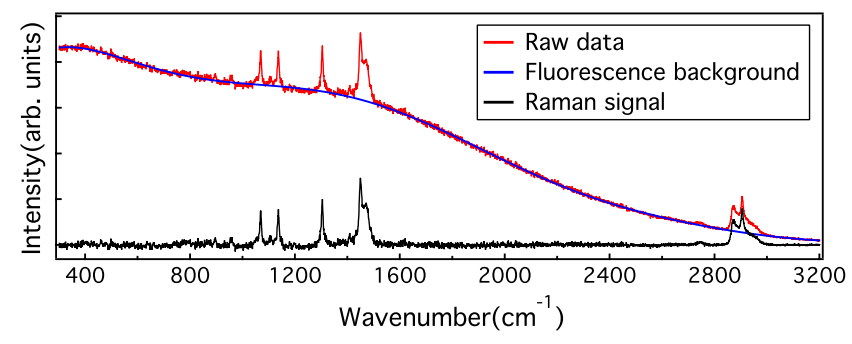

FIG. 5. The background (blue line) is a polynomial fit of the peakless region of the raw data. The black line is the residue after subtracting the background.

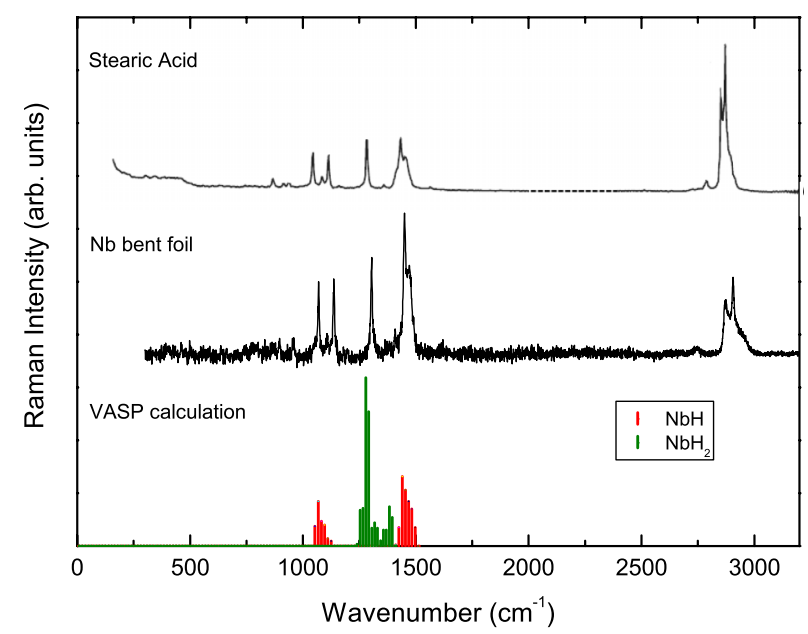

FIG. 6. Top: Raman spectrum of stearic acid [17]. Middle: Raman spectrum of a cold-worked foil stripped of background. Bottom: DFT calculations of $\mathrm{NbH}$ and $\mathrm{NbH}_{2}$ modes.

report based on limited data [31]. However, such hydride complexes do not explain the peaks near $2900 \mathrm{~cm}^{-1}$.

\section{Hydrocarbon modes}

High wave number activity may be indicative of a surface species, and particularly, $\mathrm{C}-\mathrm{H}$ stretching modes were suspected. A literature search yielded the Raman spectrum of stearic acid [17], which could explain not only the peaks near $2900 \mathrm{~cm}^{-1}$, but also several of the lower wave number peaks. For comparison with the literature spectrum, we removed the fluorescence background from the cold-worked foil spectrum of Fig. 3. The background was obtained by blocking the peak region and fitting the rest of the data with a polynomial, as shown in Fig. 5.

In Fig. 6, we compared our spectrum with that of stearic acid [17], which is a chain compound with 18 carbon atoms. The Raman peak frequencies and their assignments are shown in Table II. Additionally, we show the DFT calculations of partial DOS of the entire optical branches of $\mathrm{NbH}$ and $\mathrm{NbH}_{2}$. Since the phonon modes of $\mathrm{NbH}$ and $\mathrm{NbH}_{2}$ are near the same locations of the mid-wave-number modes of $\mathrm{CH}_{2}$, and they coincide with our measured Raman spectra, the presence of $\mathrm{NbH}$ and $\mathrm{NbH}_{2}$ cannot

TABLE II. Raman vibrational assignments and peak frequencies for rough patches on $\mathrm{Nb}$ bent foil and stearic acid [17].

\begin{tabular}{lcl}
\hline \hline Stearic acid $\left(\mathrm{cm}^{-1}\right)$ & $\mathrm{Nb}$ bent foil $\left(\mathrm{cm}^{-1}\right)$ & Assignment \\
\hline 1063 & 1068 & $\mathrm{C}-\mathrm{C}$ stretch \\
1130 & 1140 & $\mathrm{C}-\mathrm{C}$ stretch \\
1296 & 1303 & $\mathrm{C}-\mathrm{C}$ twist \\
1441 & 1450 & $\mathrm{CH}_{2}$ bend \\
1461 & 1471 & $\mathrm{CH}_{2}$ bend \\
2846 & 2870 & $\mathrm{CH}_{2}$ stretch \\
2881 & 2904 & $\mathrm{CH}_{2}$ stretch \\
\hline \hline
\end{tabular}


be ruled out [31], however the spectrum as a whole seems to be better explained by chain-type hydrocarbons.

To eliminate possible hydrocarbon contamination, Jlab hot spot samples and all recrystallized foils were subjected to an ozone treatment [33]. The treatment was performed in an atomic layer deposition system at $100^{\circ} \mathrm{C}$ with five cycles of 60 seconds ozone and 2 seconds nitrogen purge. No difference was observed in Raman spectra after the treatment. This might indicate that the sharp peaks are not due to an adsorbed $\mathrm{CH}_{2}$ layer. Furthermore, an adsorbed layer might be expected to show Raman peaks over the entire surface, but, as discussed, the Raman peaks are only observed in specific, rough pits. As another check, Fourier transform infrared (FTIR) microscopy/spectroscopy was performed on the same regions of hot spot samples and cold-worked foils and strong $\mathrm{C}-\mathrm{H}$ stretch absorptions near $2900 \mathrm{~cm}^{-1}$ were found. Since FTIR has a larger skin depth for these hydrocarbon absorptions than Raman (roughly a factor of 2), it therefore probes a bit deeper and the strong absorption again points to the hydrocarbon region occurring deeper into the surface than might be expected from an adsorbed monolayer.

Other regions of the hot spot samples and cold-worked foils revealed a different, yet still reproducible, Raman spectrum as shown in Fig. 7. These spectra do not show the sharper hydrocarbon modes but rather two broad peaks near $1350 \mathrm{~cm}^{-1}$ and $1600 \mathrm{~cm}^{-1}$ which have been identified as the D band of amorphous carbon (a-C) and $\mathrm{G}$ band of graphite respectively [34-36]. Given that the $\mathrm{G}$ band is quite broad we simply refer to these Raman spectra as a-C. We present the data from Ref. [37] in Fig. 7 where an assumed a-C layer of a few $\mathrm{nm}$ is present on top of $\mathrm{NbC}$

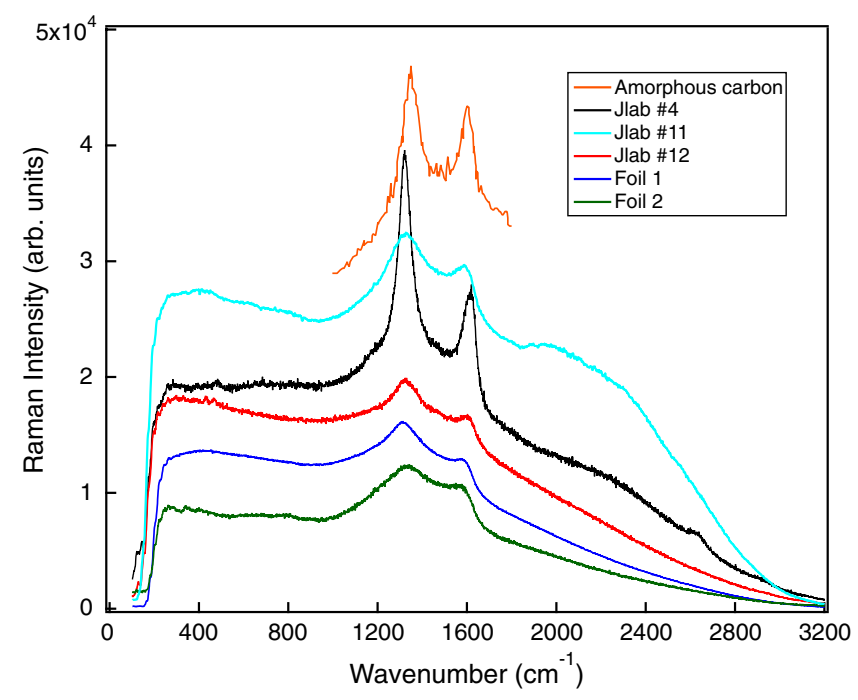

FIG. 7. Raman spectra taken from cold-worked $\mathrm{Nb}$ foils and Jlab hot spots in comparison with spectrum from amorphous carbon [37]. Foils 1 and 2 are from Alpha Aesar that have been annealed to the melting point in UHV and subsequently cold worked in air as described in the text. The legend shows the order of the spectra based on height of the $1300 \mathrm{~cm}^{-1}$ peak. nanoparticles. The stronger signal/noise observed here suggests that the a-C regions are thicker than a few nm. While a detailed analysis of these spectra is ongoing it appears that there may be varying degrees of hydrogenation of the a-C and it has been reported that the strong fluorescence background in Raman as observed in Fig. 4 is linked to $\mathrm{H}$ saturation of a-C dangling bonds [34].

The a-C modes help explain some peculiarities of Fig. 4 . While the spectrum of \#12 shows only the sharp hydrocarbon modes, other spectra such as \#3, \#9, and \#10 show the hydrocarbon peaks on top of broader peaks which appear to be the a-C modes including the $\mathrm{G}$ band near $1600 \mathrm{~cm}^{-1}$. The picture emerging is that Raman is revealing amorphous carbon regions with varying amounts of hydrogenation, including, in some cases, longer chain hydrocarbons. At this time it is not clear whether the long-chain hydrocarbons have formed as a consequence of $\mathrm{C}$ and $\mathrm{H}$ migration to dislocations along with annealing or whether compounds such as stearic acid are already present in the $\mathrm{Nb}$. While stearic acid is a common lubricant for deep drawing and is used in soaps in machining, it is difficult to understand how such molecules could withstand the significant etching and annealing (e.g. $600^{\circ} \mathrm{C}$ [11]) that is used in cavity processing.

\section{E. SEM/EDX results}

The Raman measurements prompted a reexamination of hot spot samples by SEM/EDX. On Jlab \#12, the faceted pits showed no difference in EDX spectra. This confirms earlier reports [11] and is consistent with the Raman results in Fig. 1 on sample \#9. There are other pits that look rough under SEM that showed significantly higher carbon concentration. In the left panel of Fig. 8, we show an image of
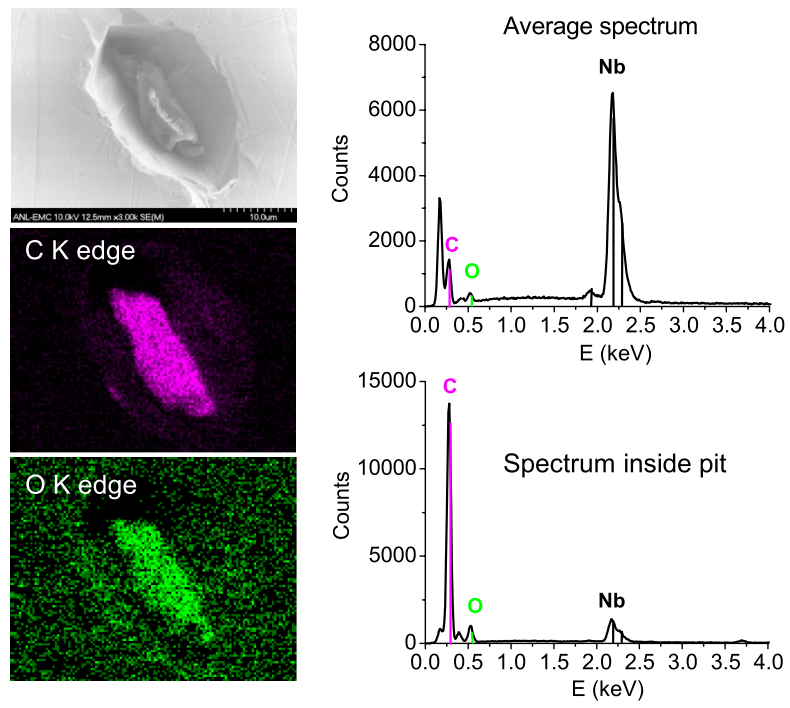

FIG. 8. Left: SEM image and EDX mapping of carbon and oxygen content of a pit on Jlab \#12. Right: An average EDX spectrum of the whole area (top) and a spectrum taken inside the pit (bottom). 


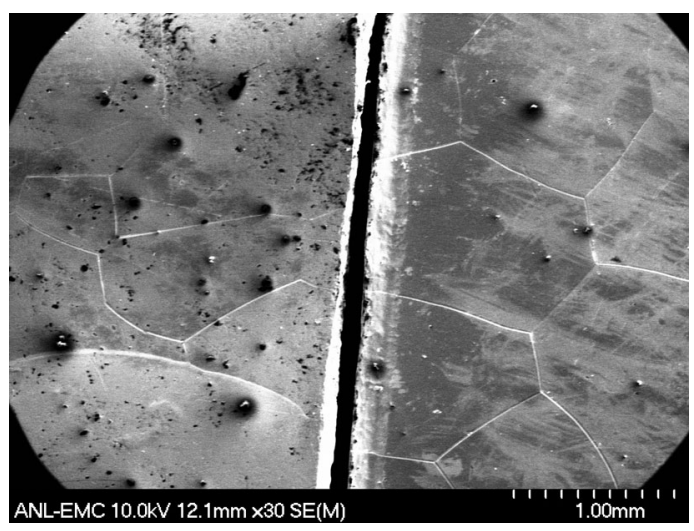

FIG. 9. SEM image of bent (left) and unbent (right) foils. Bent foil shows higher density of dark patches.

a pit that appears quite different from the smooth, shiny faceted types, along with EDX mapping of its carbon and oxygen content. The pit looks like a faceted etch pit with a rough patch of material in the center. EDX mapping shows high $\mathrm{C}$ and $\mathrm{O}$ content patches following the shape of the rough patch while the rest of the faceted pit shows little difference in $\mathrm{C}$ and $\mathrm{O}$ intensities. EDX spectra were taken from the whole region and inside of the rough patch as shown in the right panel of Fig. 8. The spectrum inside shows a dominating carbon peak. It should be noted that high carbon concentration is often accompanied by high oxygen concentration.

In Fig. 9 is shown a SEM image of bent (left) and unbent (right) foils. The bent foil shows a higher density of dark patches. An EDX mapping of $\mathrm{C}$ and $\mathrm{O}$ content of dark patches is shown in the left panel of Fig. 10. The same increase in $\mathrm{C}$ and $\mathrm{O}$ content is observed following the shape of the dark patches. The right panel shows EDX
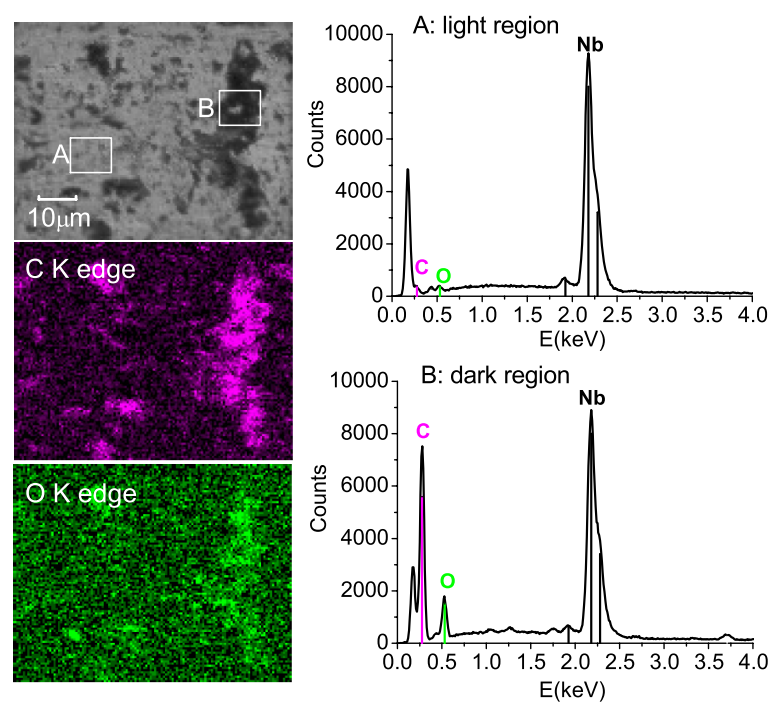

FIG. 10. Left: EDX mapping of carbon and oxygen content of a region on the bent foil. Right: EDX spectra taken in light and dark regions as indicated in the top left image.

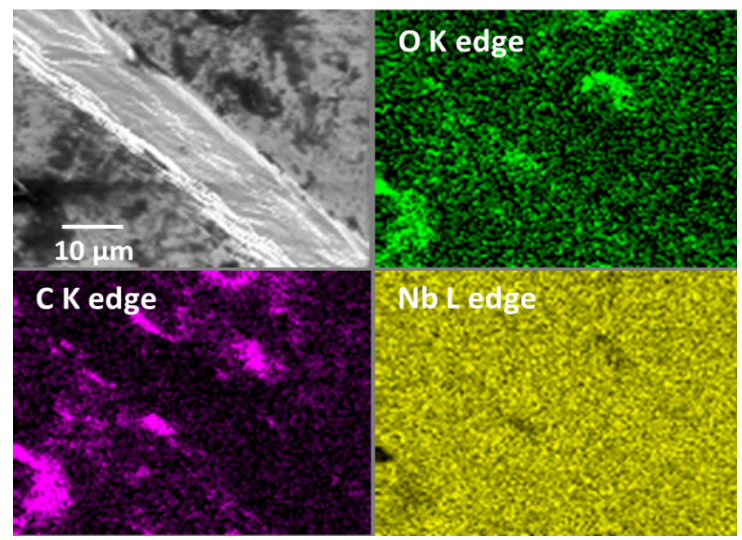

FIG. 11. EDX mapping of a region next to a grain boundary on the bent foil.

spectra taken from light and dark regions labeled A and B in the top left image.

We also did EDX mapping of a grain boundary on the bent foil as shown in Fig. 11. The grain boundary did not show any difference in chemical composition which is in agreement with the Raman results discussed earlier. Only the dark patches show higher concentration of carbon and oxygen. Some of the regions even show reduced concentration of niobium indicative of large volume of carbon compounds since the probing depth is around $1 \mu \mathrm{m}$.

\section{SUMMARY AND CONCLUSION}

Raman microscopy/spectroscopy, in conjunction with DFT calculations, has been shown to be an effective probe of the $\mathrm{Nb}$ surface, relevant for SRF cavity development. The quality of the DFT calculations has been demonstrated by the comparison between the calculated and experimental spectra of $\mathrm{Nb}$ oxide powders. A focus of this work has been to use backscatter Raman to gain insight into the correlation of high etch pit density with the formation of hot spots that lead to medium field Q drop. The Raman spectra observed in particular types of macroscopic pit defects that appear very rough under an optical microscope showed reproducible features of enhanced intensity and sharp peaks, in stark contrast with the featureless spectra of surrounding regions. Other pits which appear smooth and faceted showed no difference with the surroundings.

Comparison of the measured peaks with DFT calculations as well as with results in the literature indicated the presence of carbonaceous impurities in such rough pits. The close agreement of some peaks with calculated $\mathrm{NbH}$ and $\mathrm{NbH}_{2}$ modes indicates that these compounds cannot be ruled out, however, the overall Raman spectra, especially the high frequency modes near $2900 \mathrm{~cm}^{-1}$, more closely resemble that of $\mathrm{CH}_{2}$ chain compounds such as stearic acid. Another group of Raman spectra on hot spots and $\mathrm{Nb}$ foils showed the presence of amorphous carbon. Subsequent reexamination of the hot spot samples by 
SEM/EDX confirmed the presence of high $\mathrm{C}$ and $\mathrm{O}$ concentrations in particular macroscopic pits. Such high $\mathrm{C}$ and $\mathrm{O}$ regions sometimes occupy a significant fraction of the electron probing depth in EDX, $\sim 1 \mu \mathrm{m}$.

It is suggested that considerable migration of bulk $\mathrm{C}$ and $\mathrm{H}$ has taken place, as a consequence of the combination of strain induced dislocations and annealing. The presence of oxygen in some regions probably arises from diffusion from the surface oxide. It may be possible that impuritystabilized dislocation bundles have formed in $\mathrm{Nb}$ cavities that are resistant to disintegration by a $600^{\circ} \mathrm{C}$ bake, and lead to a high density of etch pits after acid etching. Some of these bundles might contain high concentrations of $\mathrm{C}, \mathrm{H}$, and $\mathrm{O}$ allowing the formation of $\mathrm{CH}_{2}$ chains due to dissolved hydrogen, or that the hydrocarbons were already present in the host $\mathrm{Nb}$ material. In the latter case, the presence of stearic acid (or some other fatty acid) might originate from soaps used to clean the plate or lubricants used in deep drawing. Such stable compounds might migrate to dislocations.

The connection between the $\mathrm{C}-\mathrm{H}$ modes and dislocations has been reinforced by the observation of identical Raman peaks in rough patches that were created by the cold working of previously annealed $\mathrm{Nb}$ foils. Such rough patches reveal high $\mathrm{C}$ and $\mathrm{O}$ concentrations under SEM/ EDX identical to that found in hot spot cavity samples. The picture that emerges is that regions of high dislocation density can serve as a trap of $\mathrm{C}, \mathrm{O}$, and $\mathrm{H}$ or of stable, preexisting, hydrocarbon inclusions.

The surface chemistry of such macroscopic defects, being considerably different from the bulk $\mathrm{Nb}$, must lead to reduced or nonexistent superconductivity causing localized enhanced dissipation (hot spots). It should be noted that in very early studies, surface carbon was suspected of severely limiting the accelerating fields of Nb SRF cavities [37] but the Raman studies here show that the excess carbon is quite localized. Such hot spot regions will generate thermal quasiparticles, thereby increasing the size of the hot spot, enhancing Rs and decreasing Q. The Raman work has thus provided a plausible explanation for the correlation of hot spots with the high density of etch pits. However, we note that not all etch pits are identical. The more standard-type faceted etch pits do not show enhanced carbon or hydrogen content. The distinction between these types of macroscopic pits, along with the formation of hydrocarbons, requires further investigation.

\section{ACKNOWLEDGMENTS}

This work was supported by DOE-HEP through FNAL Laboratory-University Collaboration to Understand Performance Limits of SRF Cavities. Use of the Center for Nanoscale Materials was supported by the U.S. Department of Energy, Office of Science, Office of Basic Energy Sciences, under Contract No. DE-AC0206CH11357. The electron microscopy was accomplished at the Electron Microscopy Center for Materials Research at Argonne National Laboratory, a U.S. Department of Energy Office of Science Laboratory operated under Contract No. DE-AC02-06CH11357 by UChicago Argonne, LLC. Argonne, a U.S. Department of Energy Office of Science laboratory, is operated under Contract No. DE-AC02-06CH11357. The calculations were performed at Fermi National Accelerator Laboratory, which is operated by Fermi Research Alliance, LLC under Contract No. DE-AC02-07CH11359 with the United States Department of Energy.

[1] E. Malumud,Accelerators and Beams Tools of Discovery and Innovation (APS, New York, 2010).

[2] H. Padamsee, RF Superconductivity Vol II Science, Technology and Applications (Wiley-VCH, Weinheim, 2009).

[3] E. W. Hoyt, J. Vac. Sci. Technol. 9, 144 (1972).

[4] J. Knobloch, in Hydrogen in Materials \& Vacuum Systems: First International Workshop on Hydrogen in Materials and Vacuum Systems, edited by G. R. Myneni and S. Chattopadhyay (AIP, Melville, New York, 2003), pp. 133-50.

[5] R. Ricker and G. Myneni, J. Res. Natl. Inst. Stand. Technol. 115, 353 (2010).

[6] W. Singer, X. Singer, and H. M. Wen, Materiaux Techniques 91, 13 (2003).

[7] M. Hormann, J. Less-Common Met. 139, 1 (1988).

[8] W. DeSorbo, Phys. Rev. 132, 107 (1963).

[9] N. M. Jisrawi, M. W. Ruckman, T.R. Thurston, G. Reisfeld, M. Weinert, M. Strongin, and M. Gurvitch, Phys. Rev. B 58, 6585 (1998).

[10] S. Isagawa, J. Appl. Phys. 51, 4460 (1980).

[11] X. Zhao, G. Ciovati, and T. R. Bieler, Phys. Rev. ST Accel. Beams 13, 124701 (2010).

[12] L. D. Cooley, D. Burk, C. Cooper, N. Dhanaraj, M. Foley, D. Ford, K. Gould, D. Hicks, R. Novitski, A. Romanenko, R. Schuessler, C. Thompson, and G. Wu, IEEE Trans. Appl. Supercond. 21, 2609 (2011).

[13] P. R. Evans, J. Less-Common Met. 6, 253 (1964).

[14] A. H. Cottrell, Dislocations and Plastic Flow in Crystals (Clarendon, Oxford, 1953).

[15] R. J. Cava, B. Batlogg, J. J. Krajewski, H. F. Poulsen, P. Gammel, W. F. Peck, and L. W. Rupp, Phys. Rev. B 44, 6973 (1991).

[16] T. Proslier, J. Zasadzinski, L. Cooley, C. Antoine, J. Moore, J. Norem, M. Pellin, and K. Gray, Appl. Phys. Lett. 92, 212505 (2008).

[17] P. Larkin, Infrared and Raman Spectroscopy-Principles and Spectral Interpretation (Elsevier, Amsterdam, 2011), p. 149.

[18] G. Kresse and J. Furthmuller, Phys. Rev. B 54, 11169 (1996).

[19] G. Kresse and J. Hafner, Phys. Rev. B 47, 558 (1993).

[20] J. P. Perdew, K. Burke, and M. Ernzerhof, Phys. Rev. Lett. 77, 3865 (1996).

[21] G. Kresse and D. Joubert, Phys. Rev. B 59, 1758 (1999).

[22] P. E. Blöchl, Phys. Rev. B 50, 17953 (1994). 
[23] H. J. Monkhorst and J. D. Pack, Phys. Rev. B 13, 5188 (1976).

[24] K. Parlinski, Z. Q. Li, and Y. Kawazoe, Phys. Rev. Lett. 78, 4063 (1997).

[25] K.P. Huber and G. Herzberg, Molecular Spectra and Molecular Structure. IV. Constants of Diatomic Molecules (Van Nostrand Reinhold Co., New York, 1979).

[26] T. Schober, M. Pick, and H. Wenzl, Phys. Status Solidi A 18, 175 (1973).

[27] H. Muller and K. Weymann, J. Less-Common Met. 119, 115 (1986).

[28] V. V. Sumin, Kristallografiya 34, 655 (1989).

[29] H. J. Schweizer and R. Gruehn, Z. Naturforsch., B: Anorg. Chem., Org. Chem., Biochem., Biophys., Biol. 37, 1361 (1982).

[30] B. M. Gatehouse and A. D. Wadsley, Acta Crystallogr. 17, 1545 (1964).
[31] J. Zasadzinski et al., in Proceedings of the SRF Conference (JACoW, Chicago, 2011), THPO072 [http:// epaper.kek.jp/SRF2011/papers/thpo072.pdf].

[32] F. Li and J.S. Lannin, Appl. Phys. Lett. 61, 2116 (1992).

[33] T. Momose, E. Hayasaka, K. Saitou, K. Nagayama, and S. Abe, J. Vac. Sci. Technol. A 16, 961 (1998).

[34] C. Casiraghi, A. C. Ferrari, and J. Robertson, Phys. Rev. B 72, 085401 (2005).

[35] M. A. Pimenta, G. Dresselhaus, M. S. Dresselhaus, L. G. Cancado, A. Jorio, and R. Saito, Phys. Chem. Chem. Phys. 9, 1276 (2007).

[36] S. Kodama, N. Ichikuni, K. K. Bando, T. Hara, and S. Shimazu, Appl. Catal., A 343, 25 (2008).

[37] P. Wilson, Z. D. Farkas, H. A. Hogg, and E. W. Hoyt, IEEE Trans. Nucl. Sci. 20, 104 (1973). 